\title{
Perineuronal Sulfated Proteoglycans and Dark Neurons in the Brain and Spinal Cord: A Histochemical and Electron Microscopic Study of Newborn and Adult Mice
}

\author{
Takuro Murakami, Aiji Ohtsuka, Takehito Taguchi and Da Xun Piao \\ Department of Anatomy, Okayama University School of Medicine, Okayama, Japan
}

Received June 12, 1995

\begin{abstract}
Summary. Neurons of intracerebellar nuclei in the mouse brain were demonstrated to possess a marked surface coat, formed 3-4 weeks after birth, which was stainable with cationic iron colloid or aldehyde fuchsin. Neurons with a similar surface coat were noted as relay or local interneurons in rather restricted areas such as the occipital cortex, retrosplenial cortex, zona incerta, hippocampal subiculum and spinal posterior horn. Dark neurons with condensed cytoplasm were also shown to be covered with the surface coat.

The surface coat was stained doubly with cationic iron colloid and aldehyde fuchsin. Digestion with hyaluronidase eliminated the stainability of the surface coat to both agents. Combined digestion with chondroitinase $\mathrm{ABC}$, heparitinase and keratanase eliminated the cationic iron colloid staining of the surface coat, but did not interfere with the aldehyde fuchsin staining of the surface coat.

Electron microscopy of ultrathin sections revealed that the iron particles indicating sulfated proteoglycans were preferentially deposited in the perineuronal tissue spaces. Many neurons in the hippocampal subiculum possessed cell surface glycoproteins which were labeled with lectin Vicia villosa or soybean agglutinin and formed 1-2 weeks after birth. Double staining revealed that these lectin-labeled neurons were identical in part with the neurons reactive to the cationic iron colloid.

Dark neurons began to appear 3-4 weeks after birth. The formation of perineuronal sulfated proteoglycans and the appearance of dark neurons, both occurring during the weaning period, may reflect the morphological and physiological completion of the brain. Dark neurons are suggested to be exhausted cells that are restored to light or normal neurons after sleep.
\end{abstract}

Our previous light microscopic studies of the rat brain and spinal cord stained with cationic iron colloid revealed the occurrence of numerous neurons with an intensely negatively charged surface coat (Murakami et al., 1993a, c, d). Similar neurons have been recognized also in the human brain (visual cortex) (MURAKAMI et al., 1993b) and in the brains of the cow, cat, mouse and other animals, including some lower vertebrates such as fish (MURAKAMI, 1994; MURAKAMI et al., 1994b).

Our recent histochemical and electron microscopic studies of the human visual cortex showed that the surface coat consisted of sulfated proteoglycans which were digested with hyaluronidase and chondroitinase $\mathrm{ABC} /$ heparitinase/keratanase and distributed, as the extracellular matrix, in the perineuronal tissue spaces (MURAKAMI et al., 1994a, 1995). These studies also showed that the neurons, including the dark neurons, were frequently coated with cell surface glycoproteins reactive to lectin Vicia villosa or soybean agglutinin (MURAKAMI et al., 1994a, 1995).

The present histochemical and electron microscopic reinvestigations in the mouse show that the surface coats in the brain and spinal cord of this animal, like those in the human visual cortex (MURAKAMI et al., 1994a, 1995), consist of perineuronal sulfated proteoglycans. The present study further indicates that these sulfated proteoglycans are formed 3-4 weeks after birth, and that the dark neurons begin to appear 3-4 weeks after birth. Topographic findings of the neurons with sulfated proteoglycans are also included.

\section{MATERIALS AND METHODS}

\section{Light microscopy of serial sections}

Adult (16-week-old) ICR mice, anesthetized with 
ethyl ether in the morning or in the evening, were perfused through the thoracic aorta with Ringer's solution and a mixture of $2.5 \%$ glutaraldehyde and $4 \%$ paraformaldehyde in $0.1 \mathrm{M}$ cacodylate buffer $(\mathrm{pH}$ 7.2-7.4). Immediately after this fixation, the brain and cervical spinal cord were isolated.

The isolated tissues were sliced into $2-3 \mathrm{~mm}$-thick blocks along a frontal plane, and refixed in the buffered glutaraldehyde/paraformaldehyde fixative for $6 \mathrm{~h}$ or longer. The blocks were embedded in paraffin, and cut into serial sections along the frontal plane. Every ten sections of these serial sections were deparaffinized with xylene.

The deparaffinized sections were incubated in cationic iron colloid with $\mathrm{pH}$ values of $1.0-1.5$, immersed in a mixture of $\mathrm{K}_{4} \mathrm{Fe}(\mathrm{CN})_{6}$ and $\mathrm{HCl}$ for Prussian blue reaction, counterstained with carbol thionin or nuclear fast red, and observed with a light microsope (MURAKAMI et al., 1986, 1993a).

\section{Light microscopic and histochemical studies}

Neonatal (1-2 weeks after birth), young (3-5 weeks after birth), pubescent (6-8 weeks after birth) and adult (15-16 weeks after birth) ICR mice were sacrificed under ether anesthesia in the afternoon or evening. From these animals, small 2-3 mm-thick blocks traversing the hippocampal subiculum or intracerebellar nuclei along a frontal plane were removed. The blocks were fixed with $4 \%$ paraformaldehyde in $0.1 \mathrm{M}$ cacodylate buffer ( $\mathrm{pH}$ 7.2-7.4) for $6 \mathrm{~h}$ or longer, embedded in paraffin, and cut into sections along the frontal plane. These sections were deparaffinized with xylene.

The deparaffinized sections were stained with cationic iron colloid at $\mathrm{pH}$ values of 1.0-1.5 (MURAKAMI et al., 1986), aldehyde fuchsin (GOMORI, 1950; MURAKAMI et al., 1994a), lectin Vicia villosa agglutinin (VVA) (NAKAGAWA et al., 1986; MURAKAMI et al., 1994a) or lectin Glycine max (soybean) agglutinin (SBA) (MURAKAMI et al., 1995). Some sections were stained doubly with cationic iron colloid and aldehyde fuchsin (MURAKAMI et al., 1995). Furthermore, some sections were digested solely with hyaluronidase or successively with chondroitinase ABC, heparitinase and keratanase prior to the staining with the cationic iron colloid, aldehyde fuchsin or lectins (MURAKAMI et al., 1994d).

\section{Electron microscopic study}

Adult ICR (15-16 weeks after birth) mice were fixed in the morning under ether anesthesia by vascular perfusion with a mixture of $2.5 \%$ glutaraldehyde and $4 \%$ paraformaldehyde in $0.1 \mathrm{M}$ cacodylate buffer $(\mathrm{pH}$ 7.2). Small 2-3 mm-thick blocks containing the hippocampal subiculum were isolated. They were cut into smaller blocks $(1 \times 2 \times 2 \mathrm{~mm})$, and fixed again for
$6 \mathrm{~h}$ in the buffered paraformaldehyde/glutaraldehyde mixture. The blocks were embedded in LR White resin, and cut into ultrathin sections. These sections were incubated in cationic iron colloid with $\mathrm{pH}$ values of 1.0-1.5, exposed to osmium vapor, and observed with a transmission electron microscope (OHTSUKA et al., 1993).

\section{RESULTS}

\section{Light microscopy of serial sections}

Survey light microscopy of every 10 samples from the serial sections revealed that the neurons with intensely negatively charged surface coats preferentially occurred in the retrosplenial cortex, visual or occipital cortex, temporal cortex, zona incerta, red nucleus, tegmental nuclei, inferior colliculus, periolivary nuclei, hippocampal subiculum, intracerebellar nuclei and spinal posterior horn. In contrast, the olfactory bulb, frontal cortex, substantia nigra, insular cortex, putamen, claustrum, hypothalamus, dorsal column nuclei and cerebellar cortex contained few such coated neurons.

The neurons with the negatively charged coat were frequently observed in the intracerebellar nuclei and hippocampal subiculum (Fig. 1). In the intracerebellar nuclei, almost all neurons, including the small-sized Golgi's Type-II local interneurons, were reactive to the cationic iron colloid. In the hippocampal subiculum, an average of 3 out of 10 neurons possessed the surface coat. The surface coats in these areas were well developed, and clearly observed as fine meshworks which surrounded the cell body and foot processes (Fig. 1 Upper Inset). The surface coats surrounding the dark neurons protruded many hairy projections (Fig. 1 Lower Inset). The dark neurons were characterized by their markedly shrunken cell body and condensed cytoplasm, which was intensely stained with nuclear fast red or carbol thionin (Fig. 1, Lower Inset). The dark neurons were also characterized by their nucleoplasm which was strongly reactive to nuclear fast red (Fig. 1 Lower Inset).

Few dark neurons were recognizable in the specimens prepared in the morning when the amimals were in slumber or just after waking (Fig. 2). However, many dark neurons were observed in the specimens prepared in the evening when the animals were awake and actively eating and drinking (Fig. 1).

In this respect, the hippocampal subiculum and intracerebeller nuclei were well exemplified. Six to eight of 50 neurons with or without an intensely negatively charged surface coat (see above) were 

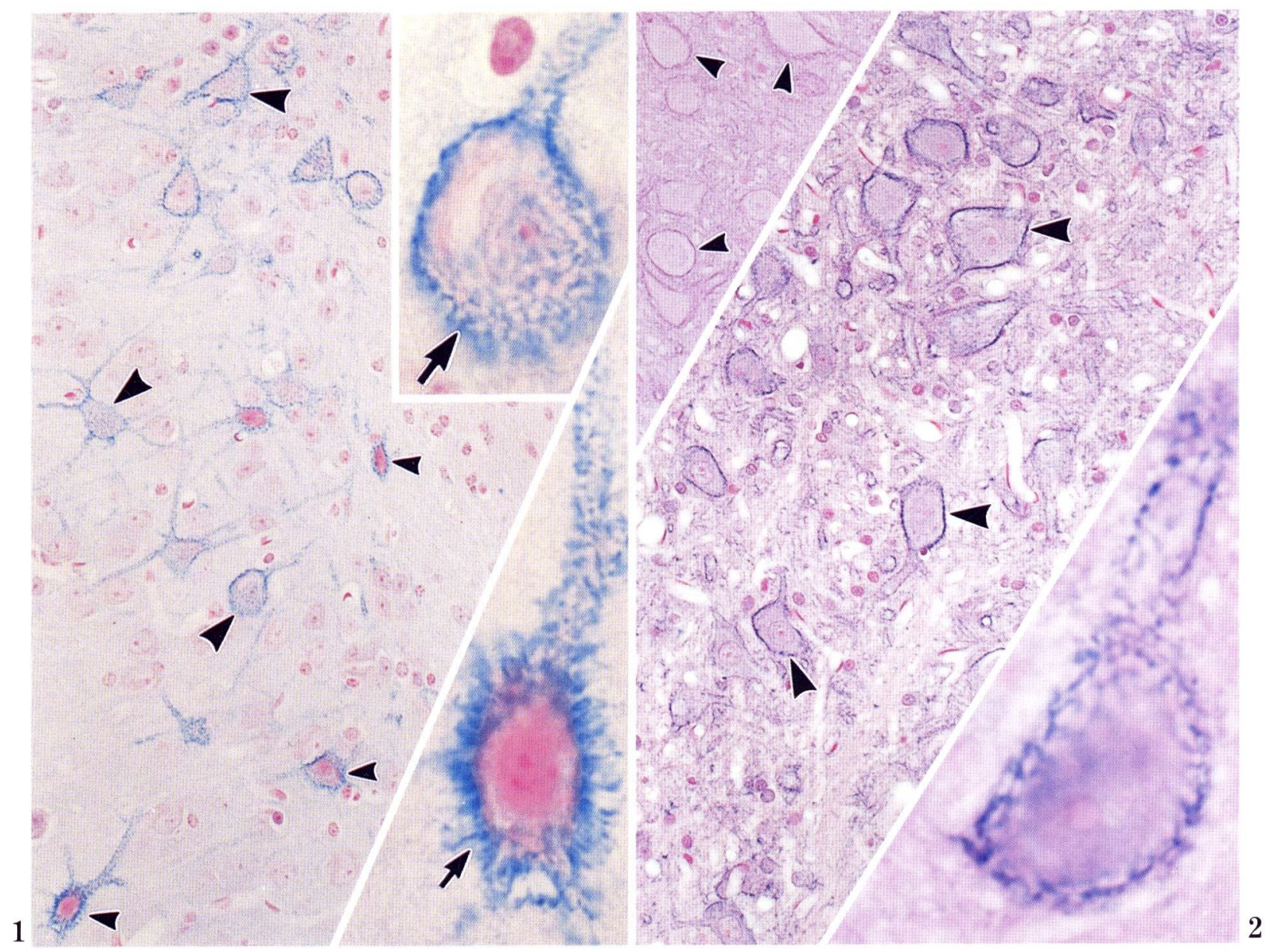

Fig. 1. Survey light micrograph of an adult mouse brain section traversing the hippocampal subiculum along a frontal plane, stained with cationic iron colloid ( $\mathrm{pH}$ 1.5) and nuclear fast red. Many neurons (thick arrowheads), including some dark neurons (thin arrowheads), possess marked surface coats which are reactive to cationic iron colloid. This specimen was obtained from an animal sacrificed at 7 o'clock in the evening. Upper inset shows the meshwork of a surface coat (large arrow) surrounding the lowermost neuron indicated by a thick arrowhead in the main figure. Lower inset shows a surface coat with hairy projections (small arrow) surrounding the lowermost dark neuron indicated by a thin arrowhead in the main figure. $\times 260$, Upper and lower insets: $\times 1,000$

Fig. 2. Medial intracerebellar nucleus as doubly stained with aldehyde fuchsin and cationic iron colloid and counterstained with nuclear fast red. Almost all neurons in this nucleus possess a surface coat reactive to both agents (thick arrowheads). Few dark neurons are seen. From a sleeping animal at 11 o'clock in the morning. Upper inset shows neurons in the medial intracerebellar nucleus as stained solely with aldehyde fuchsin (thin arrowheads). Lower inset shows the surface meshwork of a neuron (small-sized, Golgi's Type II interneuron) in the intracerebellar nuclei as doubly stained with aldehyde fuchsin and cationic iron colloid. $\times 300$, Upper inset: $\times 260$, Lower inset: $\times 1,200$

noted as dark neurons in the hippocampal specimens prepared at 7 o'clock in the evening (Fig. 1); 13-17 of 50 neurons were noted as dark neurons at 11 o'clock at night. In the intracerebellar nuclei where almost all neurons were provided with the surface coat, 1520 of 50 neurons were noted as dark neurons at 7 o'clock in the evening; 40-45 of 50 neurons were changed into dark neurons at 11 o'clock at night; few dark neurons were noted at 10 o'clock in the morning (Fig. 2).

\section{Light microscopic and histochemical studies}

Additional preparations of sections traversing the 

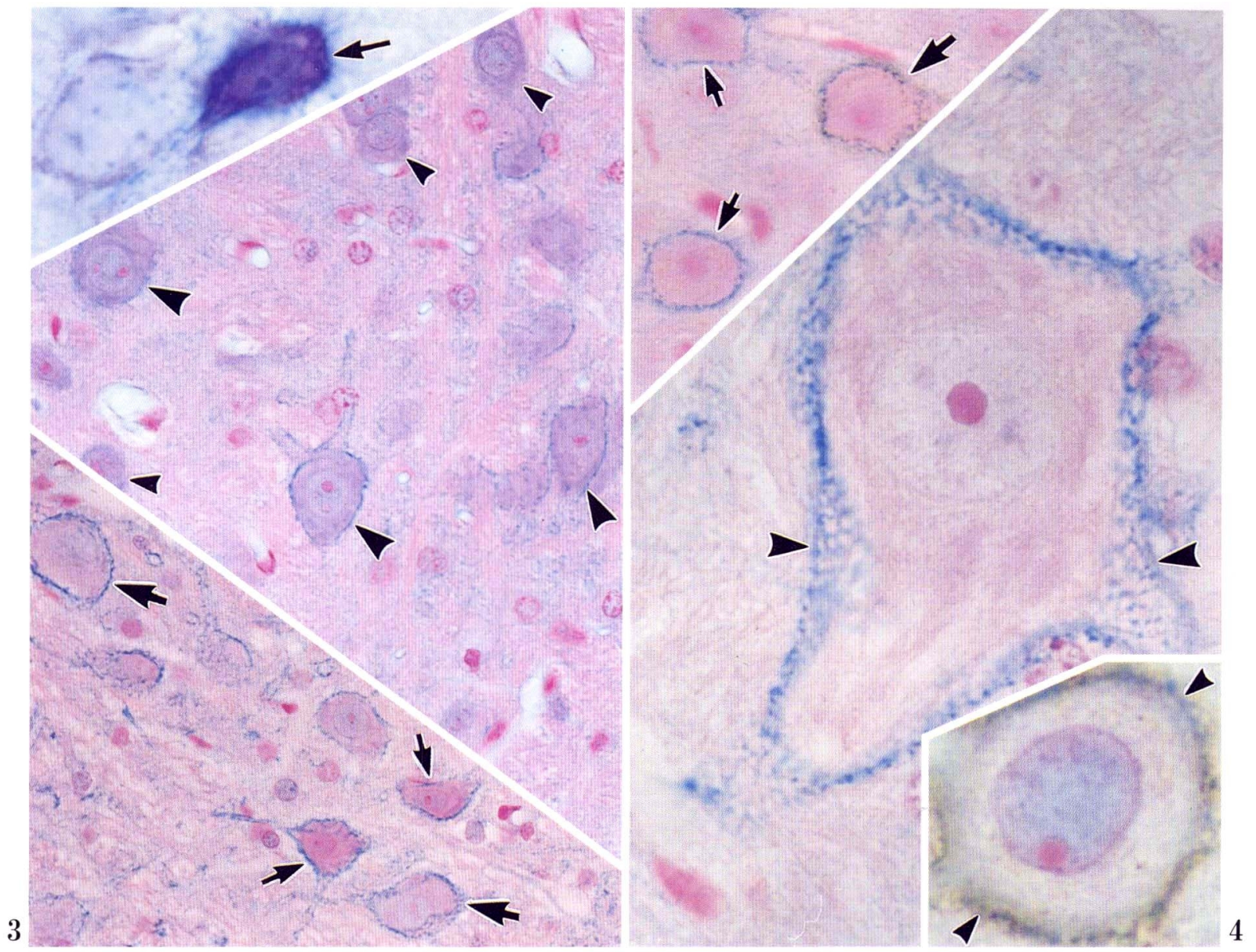

Fig. 3. Medial intracerebellar nucleus of a young mouse at 3 weeks after birth, stained with cationic iron colloid and nuclear fast red. Some neurons are slightly reactive to the colloid (thick arrowheads), while others remain unstained (thin arrowheads). Upper inset shows a dark neuron (small arrow), which was observed in the hippocampal subiculum of another young animal (3 weeks after birth) and counterstained with carbol thionin. This dark neuron is reactive to the cationic iron colloid. Lower inset shows the medial intracerebellar nucleus of a young mouse, 4 weeks after birth. Note in this inset that almost all neurons are reactive to the cationic iron colloid (large and small arrows). Small arrows indicate dark neurons. $\times 500$, Upper inset: $\times 800$, Lower inset: $\times 420$

Fig. 4. Surface meshworks (arrowheads) surrounding a neuron in the medial intracerebellar nucleus of a young mouse, 4 weeks after birth. Upper inset shows a doubly lectin VVA- and cationic iron colloid-incubated section of the hippocampal subiculum, obtained from a young mouse, 4 weeks after birth. Note that the neuron indicated by the large arrow is doubly stained with lectin and colloid, while those indicated by small arrows are solely stained with the colloid. Lower inset shows a closer view of the doubly stained surface meshwork (thin arrowheads), as observed in the hippocampal subiculum of a young mouse, 4 weeks after birth. $\times 1,000$, Upper inset: $\times 600$, Lower inset: $\times 850$

hippocampal subiculum or intracerebellar nuclei were useful for the histochemical and electron microscopic studies since these sections contained many neurons with well developed surface coats (see above). The histochemical and electron microscopic findings thus obtained are described below.

The surface coats-including those surrounding the dark neurons - in the hippocampal subiculum and intracerebellar nuclei in the adult mouse were stained with aldehyde fuchsin (Fig. 2 Upper Inset), and stained doubly with cationic iron colloid and aldehyde fuchsin (Fig. 2). The doubly stained surface coats were observed as fine meshworks (Fig. 2 Lower Inset). The double-stained surface coats surrounding the dark 

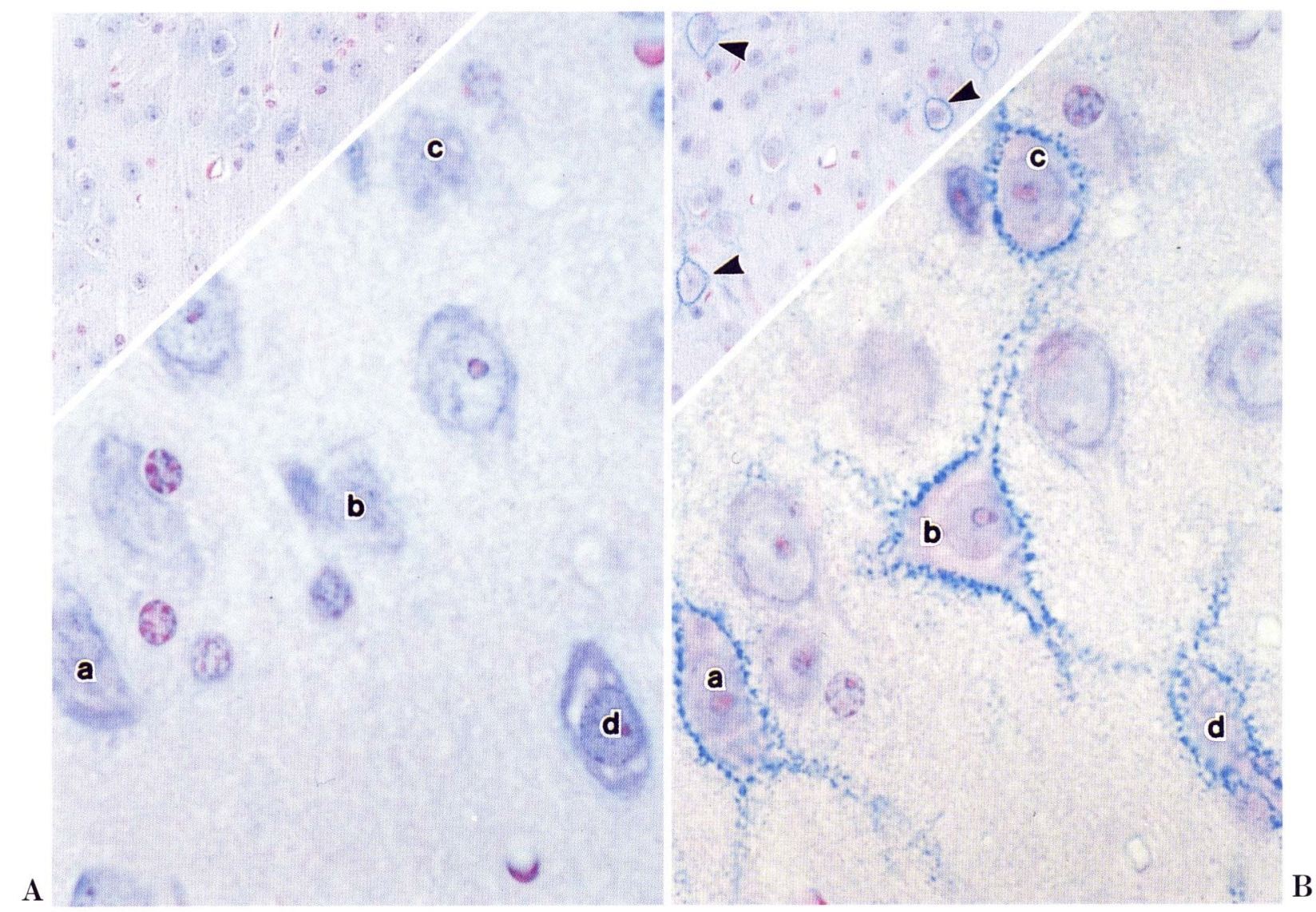

Fig. 5 A. Hippocampal section of an adult mouse (16 weeks of age), treated with hyaluronidase and stained with cationic iron colloid. No cell is reactive to the colloid. $\mathbf{B}$ shows an adjacent control section incubated in a buffer substrate without hyaluronidase and stained with cationic iron colloid. Many cells are reactive to the colloid. The neurons indicated $a-d$ in $\mathbf{A}$ are identical to those in $\mathbf{B}$, respectively. Inset in $\mathbf{A}$ shows an intracerebellar section from a young (5-week-old) mouse, treated with chondroitinase $\mathrm{ABC} /$ heparitinase/keratanase and cationic iron colloid. Note that no neuron is reactive to the cationic iron colloid. Inset in $\mathbf{B}$ shows an adjacent control section incubated in a buffered substrate lacking in the enzyme and stained with cationic iron colloid. Many neurons are shown reactive to the cationic iron colloid (arrowheads). A and B: $\times 700$, Insets in A and B: $\times 220$

neurons, like those solely stained with cationic iron colloid, protruded many hairy projections.

The intracerebellar nuclei in the adult mouse contained few neurons reactive to lectin VVA or SBA. However, the hippocampal subiculum contained many neurons whose external cell surfaces were labeled with lectin VVA or SBA. Double staining with lectin and cationic iron colloid revealed that the neurons labeled with lectin VVA or SBA were identical in part with the neurons reactive to cationic iron colloid. More strictly, 2 of 10 neurons in the hippocampal subiculum were both reactive to cationic iron colloid and lectin VVA or SBA on the average; 1 of 10 neurons were solely reactive either to cationic iron colloid or lectin VVA or SBA on average.
The hippocampal subiculum and intracerebellar nuclei of the neonatal mice at 1-2 weeks after birth contained no neuron reactive to cationic iron colloid or aldehyde fuchsin.

The surface coats became detectable in young mice at 3 weeks after birth (Fig. 3). At this stage, the surface coats were so thin that they were stained slightly with cationic iron colloid, aldehyde fuchsin or both (Fig. 3).

The surface coats rapidly developed at 3-4 weeks after birth. Therefore, at 4 weeks after birth, almost all neurons in the intracerebellar nuclei were provided with a surface coat which was clearly stainable with cationic iron colloid (Fig. 3 Lower Inset). Even in the hippocampal subiculum, 3 of 10 neurons possessed 


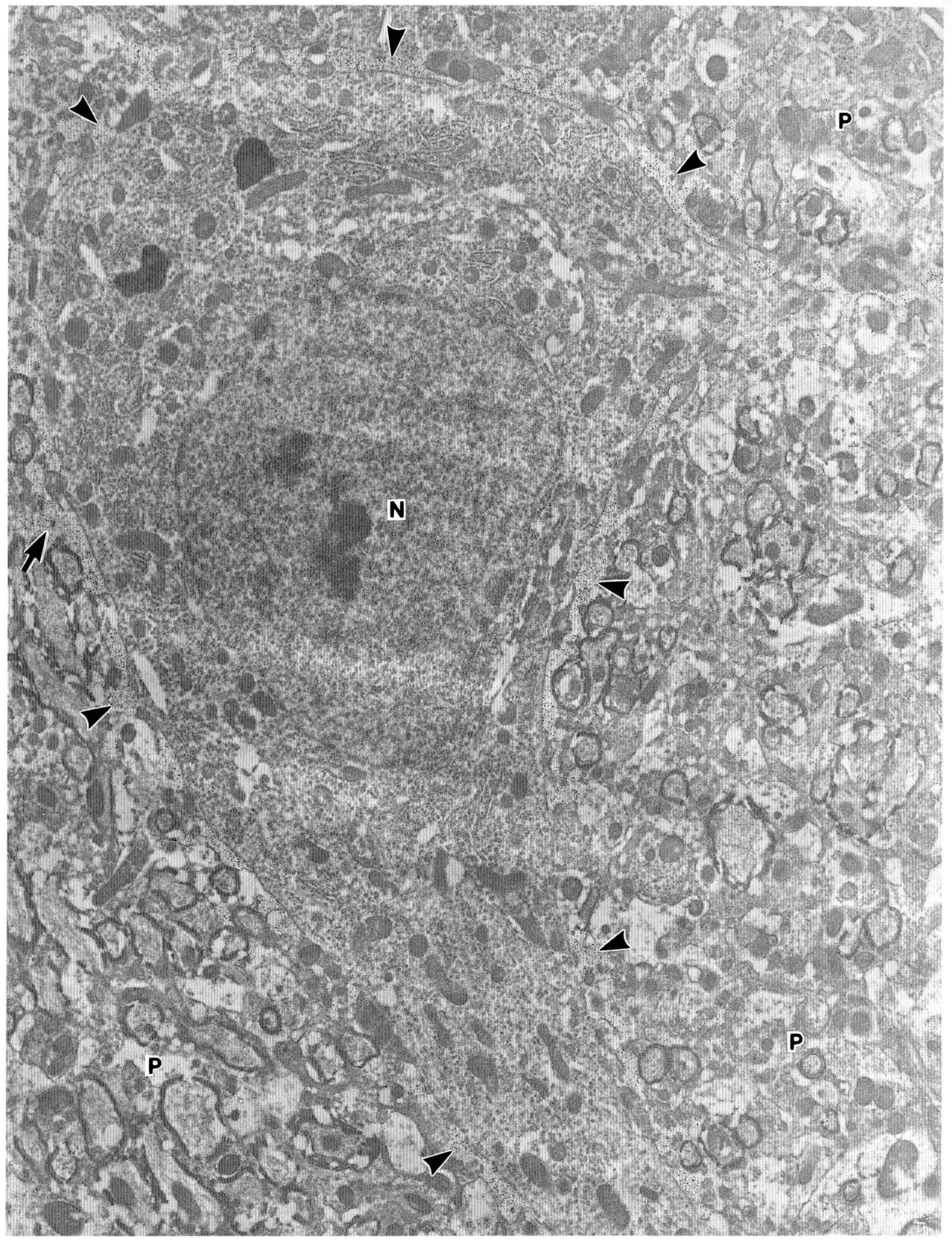

Fig. 6. Electron micrograph of a neuron $(N)$ in the hippocampal subiculum of an adult (16-week-old) mouse, stained with cationic iron colloid at $\mathrm{pH} 1.0$ and exposed to osmium vapor. The perineuronal spaces of this neuron are markedly wide and show dense depositions of iron particles (arrowheads). $P$ neuropil region. $\times 10,000$ 
the surface coat on average (Fig. 3 Upper Inset). This frequency of occurrence roughly corresponded to that in the hippocampal subiculum of the adult animal. The surface coats at this stage were represented by fine meshworks stained with cationic iron colloid as well as aldehyde fuchsin (Fig. 4).

In accordance with the findings of the adult mouse, few neurons in the intracerebellar nuclei were reactive to lectin VVA or SBA at any growth stages of the mice. In the hippocampal subiculum, however, many neurons were reactive to these lectins. Here, the lectin-VVA labeling began at 1 week after birth, whereas the lectin-SBA labeling commenced at 2 weeks after birth. The double staining at 4 weeks after birth showed that the lectin VVA- or SBAlabeled neurons were identical in part with the neurons reactive to the cationic iron colloid (Fig. 4 Upper and Lower Insets). At this stage, thus, 2 of 10 neurons in the hippocampal subiculunm were reactive to cationic iron colloid and lectin VVA or SBA. This occurrence is almost the same as that in the adult mouse (see above).

The dark neurons began to appear at 3-4 weeks after birth (Fig. 3). In the hippocampal subiculum at this stage, only 2 of 50 neurons were observed as dark neurons on average. The frequency of occurrence of dark neurons was inconsistent, though a general tendency for its increase with the growth of the animals was recognized. The dark neurons in these developing animals were also frequently provided with a surface coat reactive to cationic iron colloid (Fig. 3 Upper and Lower Insets) and labeled with lectin VVA or SBA. No dark neuron was noted at the stages from 1-2 weeks after birth.

The findings obtained from the enzyme digestions were invariable among all the specimens from the neonatal, young, pubescent and adult mice. Thus, the hyaluronidase digestion constantly eliminated both the cationic iron colloid and aldehyde fuchsin stainings of the surface coats in the growing and adult mice (Fig. 5 A, B). Another constant finding among the animals examined was that successive chondroitinase $\mathrm{ABC}$, heparitinase and keratanase treatments eliminated the cationic iron colloid staining of the surface coats (Fig. 5 A Inset, B Inset), but did not interfere with the aldehyde fuchsin staining of the surface coats. Furthermore, in all the animals examined, the hyaluronidase and chondroitinase $\mathrm{ABC} /$ heparitinase/keratanase digestions never obliterated the labelings of neurons with lectin VVA and SBA.

\section{Transmission electron microscopy}

Transmission electron microscopy of tissue sections

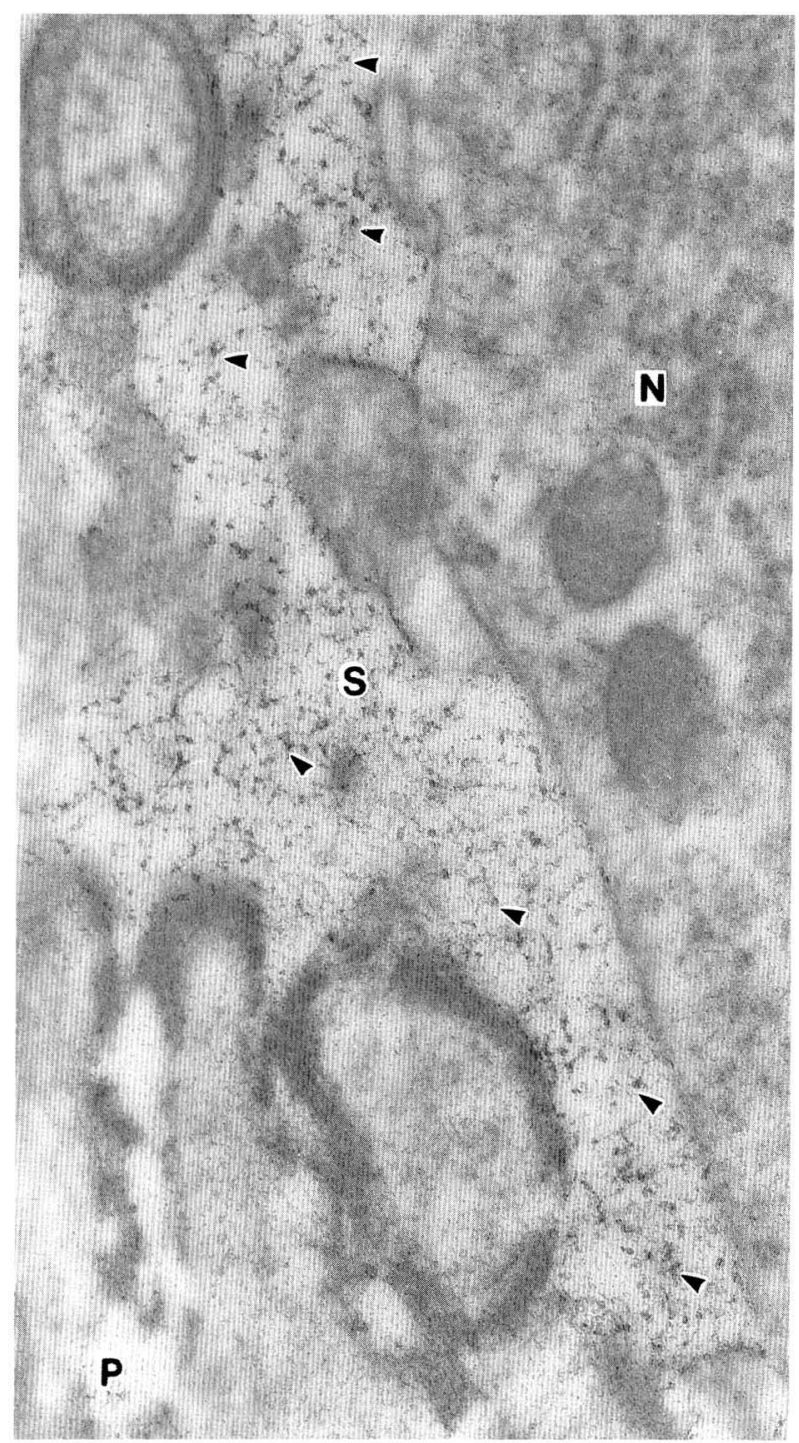

Fig. 7. Closer view of a perineuronal space as indicated by an arrow in Figure 6. Clustered iron particles (arrowheads) are diffusely deposited in the perineuronal space (S). $N$ neuronal soma, $P$ neuropil region. $\times 70,000$

from the hippocampal subiculum of the adult mouse showed that the images produced by a single staining with osmium vapor were so unclear that such fine tissue structures as the forms and sizes of the presynaptic vesicles were only obscurely contrasted. It was, nevertheless, confirmed that the perineuronal tissue spaces of certain neurons were extremely widened, ranging from $0.1-0.2 \mu \mathrm{m}$ in width, and that these spaces specially showed a preferential deposition of cationic iron colloid particles (Figs. 6, 7). Such clustered iron deposits were neither noted within the usual perineuronal tissue spaces with $10-25 \mathrm{~nm}$ in 
width nor within the neuronal somata and processes or the neuropil regions, except for insignificant solitary deposits of iron particles.

\section{DISCUSSION}

The present study confirms that the mouse brain and spinal cord contain numerous neurons with intensely negatively charged surface coats which are stained with cationic iron colloid. It also shows that these neurons preferentially occur as relay or local interneurons in rather restricted areas such as the intracerebellar nuclei and hippocampal subiculum, and that they never occur in the frontal cortex, olfactory bulb or cerebellar cortex.

The present study, together with our recent studies of the human visual cortex (MURAKAMI et al., 1994a, 1995), demonstrates that the surface coats are stained doubly with cationic iron colloid and aldehyde fuchsin, and digested by hyaluronidase and chondroitinase $\mathrm{ABC} /$ heparitinase/keratanase, these findings indicating that the surface coats consist of sulfated proteoglycans. Similar surface coats or meshwork structures have been shown in the rat and mouse with Golgi's silver impregnation, and interpreted as representing the processes of glial cells (glial nets) (BRAUER et al., 1982; 1984; LÜTH et al., 1992; CELIO and BLÜMCKE, 1994).

The present study demonstrates that the perineuronal sulfated proteoglycans are formed at postnatal stages 3-4 weeks after birth. These stages correspond to the weaning period of the animals (see below).

The present study reveals that the mouse hippocampal subiculum contains many neurons which are labeled with lectin VVA or SBA, and that these lectin labelings begin at the postnatal stages of 1-2 weeks after birth. Thus, the cell surface glycoproteins reactive to lectin VVA or SBA are formed independently at earlier stages than the perineuronal sulfated proteoglycans.

The present study demonstrates that almost all neurons in the intracerebellar nuclei are reactive to cationic iron colloid or aldehyde fuchsin, but are not labeled with lectin VVA or SBA. It also demonstrates that, in the hippocampal subiculum of the growing and adult mice, lectin-labeled neurons are not always reactive to cationic iron colloid. These findings, together with those from the human visual cortex (MURAKAMI et al., 1994a, 1995), indicate that cell surface glycoproteins as labeled with lectin VVA or SBA are neither structural elements nor adhesive molecules of the sulfated proteoglycans.
The present study shows that in the mouse, the dark cells begin to appear at the weaning period, i.e., 3-4 weeks after birth, and that even the dark cells frequently possess perineuronal sulfated proteoglycans. Such hairy projections of the surface coats as observed in the dark neurons may be formed by the extraordinary shrinkage of these cells.

Recent physiological experiments of the rat brain have shown that the dark neurons are neither degenerative nor poorly fixed cells, and that the appearance of dark neurons is a reversible or plastic change induced by anodal polarization (ISLAM et al., 1994). The present study supports our view that the dark neurons are exhausted or tired cells, which are restored to normal or light neurons in the morning or after sleep or resting (MURAKAMI and OHTSUKA, 1995). Our concomitant experiments in the present study have more clearly revealed that the dark neurons in the mouse show a diurnal precision or circadian rhythm in their occurrence, and that they are not reactive to apoptotic DNA labeling or terminal deoxynucleotidyl transferase-mediated deoxyuridine triphosphate-biotin nick end labeling. The details of these findings will be reported elsewhere. Our recent discussion suggests that in the dark neurons with nucleoplasm strongly reactive to nuclear fast red, mRNA synthesis is markedly activated (MURAKAMI and OHTSUKA, 1995).

The present transmission electron microscopic observations of ultrathin sections from the adult mouse hippocampal subiculum reveal that the cationic iron colloid is preferentially and diffusely deposited in the widened perineuronal spaces. Similar findings have been obtained in the human visual cortex (MURAKAMI et al., 1994). These facts indicate that the perineuronal sulfated proteoglycans thickly surround-as an extracellular matrix - the axosomatic synapses and assure a stable signal transmission at these synapses with their inhibitory nature (MURAKAMI et al., 1994a, 1995). Our recent personal discussion with Prof. Chizuka IDE (Kyoto University) has further elicited the idea that such thick and intensely negatively charged perineuronal proteoglycans might prohibit the new formation of synapses with adjacent sprouting neuronal terminals.

Some authors have shown that the neurons labeled with lectin VVA or SBA are GABAergic interneurons (KoSAKA and HeizMAnN, 1989; DraKe et al., 1991; LÜTH et al., 1992). These data suggest that such neurons both reactive to cationic iron colloid and lectin VVA or SBA as observed in the hippocampal subiculum are inhibitory interneurons. We consider that such neurons solely reactive to cationic iron colloid as mainly observed in the intracerebellar cortex are excitatory interneurons. 
It is noteworthy that the perineuronal sulfated proteoglycans are formed at the weaning period, and that also at this period the dark or tired neurons begin to appear. The appearance of perineuronal sulfated proteoglycans as well as dark neurons thus seems to be an initial sign of brain plasticities encoding memory or learning for feeding or other actions, including volitional movements. In other words, weaning coincides with the morphological and physiological completion (maturation) of the brain.

Acknowledgments. We are grateful to Prof. Chizuka IDE (Department of Anatomy, Kyoto University Faculty of Medicine) and Prof. Shigeru Kobayashi (Department of Anatomy, Nagoya University School of Medicine) for helpful discussions. We are also grateful to Mr. Hiromichi KUSANO and Mr. Masahiro NARASAKI (technical experts in our department) for their help in tissue preparation and staining.

\section{REFERENCES}

Brauer, K., L. Werner and L. Leibnitz: Perineuronal nets of glia. J. Hirnforsch. 23: 701-708 (1982).

Brauer, K., G. BRüCKNER, L. LeIBNITZ and L. WeINER: Structural and cytochemical features of perineuronal glial nets in the rat brain. Acta Histochem. 74: 53-60 (1984).

Celio, M. R. and I. BlüMcke: Perineuronal nets. A specialized form of extracellular matrix in the adult nervous system. Brain Res. Rev. 19: 128-145 (1994).

Drake, C. T., K. A. Mulligan, T. L. Wimpey, A. Hendrickson and C. Chavkin: Characterization of Vicia villosa agglutinin-labeled GABAergic interneurons in the hippocampal formation and in acutely dissociated hippocampus. Brain Res. 554: 176-185 (1991).

Gomori, G.: Aldehyde-fuchsin: a new stain for elastic tissue. Amer. J. Clin. Pathol. 20: 665-666 (1950).

Islam, N., A. Moriwaki, Y. Hattori and Y. HoRI: Appearance of dark neurons following anodal polarization in the rat. Acta Med. Okayama 48: 123-130 (1994).

Kosaka, T. and C. W. Heizmann: Selective staining of a population of parvalbumin-containing GABAergic neurons in the rat cerebral cortex by lectins with specific affinity for terminal $\mathrm{N}$-acetylgalactosamine. Brain Res. 483: 158-163 (1989).

LÜTH, H.-J., J. Fischer and M. R. Celio: Soybean lectin binding neurons in the visual cortex of the rat contain parvalbumin and are covered by glial nets. J. Neurocytol. 21: 211-221 (1992).

MURAKAMI, T.: Strongly negatively charged extracellular matrix in the mouse central nervous system (Japanese text with English abstract). Okayama Igakkai Z. 106: 1063-1071 (1994).

Murakami, T. and A. Ohtsuka: Sleep restores dark neurons to light neurons in the central nervous system (Japanese text with English abstract). Okayama Igakkai Z. 107: (1995, in press).
Murakami, T., T. Taguchi, A. Ohtsuka, K. Sano, T. Kaneshige, R. L. Owen and A. L. Jones: A modified method of fine-granular cationic iron colloid preparation: its use in light and electron microscopic detection of anionic sites in the rat kidney glomerulus and certain other tissues. Arch. Histol. Jap. 49: 13-23 (1986).

Murakami, T., T. Taguchi, A. Ohtsuka and A. Kikuta: Neurons with strongly negative-charged surface-coats in adult rat brain as detected by staining with cationic iron colloid. Arch. Histol. Cytol. 56: 13-21 (1993a).

Murakami, T., T. Taguchi and A. Ohtsuka: The occurrence in the human brain of neurons with strongly negative-charged proteoglycans. Arch. Histol. Cytol. 56: 23-26 (1993b).

Murakami, T., T. Taguchi and A. Ohtsuka: Central nervous cells with strongly negatively-charged surfacecoats (Japanese text with English abstract). Okayama Igakkai Z. 105: 49-53 (1993c).

Murakami, T., Y. Tsubouchi, M. Tsubouchi, A. OHTSUKa, T. TAgUChI and A. KIKUTA: The ocurrence of rat spinal cord neurons with strongly negative-charged surfacecoats. Arch. Histol. Cytol. 56: 501-504 (1993d).

Murakami, T., A. Ohtsuka and T. Taguchi: Neurons with intensely negatively charged extracellular matrix in the human visual cortex. Arch Histol. Cytol. 57: 509522 (1994a).

Murakami, T., M. Tsubouchi, Y. Tsubouchi, T. Tagu CHI and A. OHTSUKa: The occurrence of neurons with strongly negatively charged surface coats in mammalian, avian, reptilian, amphibian and piscine brains. Acta Med. Okayama 48: 195-197 (1994b).

Murakami, T., S. Hitomi, A. Ohtsuka and T. Taguchi: Neurons with perineuronal sulfated proteoglycans in the human visual cortex, with special reference to their reactions to lectins. Arch. Histol. Cytol. 58: 357-364 (1995).

Nakagawa, F., A. Schulte and S. S. Spicer: Selective cytochemical demonstration of glycoconjugate-containing terminal $\mathrm{N}$-acetylgalactosamine on some brain neurons. J. Comp. Neurol. 243: 280-290 (1986).

Ohtsuka, A., A. Kinuta, T. Taguchi and T. Murakami: A hydrophilic resin-embedding method for light and electron microscopic detection of tissue anionic sites with cationic colloidal iron: as applied to mouse Paneth cells. Arch. Histol. Cytol. 56: 423-430 (1993).

Prof. Takuro Murakami

Section of Human Morphology

Department of Anatomy

Okayama University School of Medicine

2-5-1 Shikata-cho, Okayama

700 Japan

村上 宅 郎

700 岡山市鹿田町 2-5-1

岡山大学医学部

解剖学第二講座 